\title{
Optical Path Length Calibration: A Standard Approach for Use in Absorption Cell-Based IR-Spectrometric Gas Analysis
}

\author{
Javis Anyangwe Nwaboh, Oliver Witzel, Andrea Pogány, Olav Werhahn, and Volker Ebert
}

Physikalisch-Technische Bundesanstalt, Bundesallee 100, 38116 Braunschweig, Germany

Correspondence should be addressed to Olav Werhahn; olav.werhahn@ptb.de

Received 14 March 2014; Accepted 28 May 2014; Published 17 July 2014

Academic Editor: Rolf W. Berg

Copyright (C) 2014 Javis Anyangwe Nwaboh et al. This is an open access article distributed under the Creative Commons Attribution License, which permits unrestricted use, distribution, and reproduction in any medium, provided the original work is properly cited.

\begin{abstract}
We employed a comparison method to determine the optical path length of gas cells which can be used in spectroscopic setup based on laser absorption spectroscopy or FTIR. The method is based on absorption spectroscopy itself. A reference gas cell, whose length is a priori known and desirably traceable to the international system of units (SI), and a gas mixture are used to calibrate the path length of a cell under test. By comparing spectra derived from pressure-dependent measurements on the two cells, the path length of the gas cell under test is determined. The method relies neither on the knowledge of the gas concentration nor on the line strength parameter of the probed transition which is very rarely traceable to the SI and of which the uncertainty is often relatively large. The method is flexible such that any infrared light source and infrared active molecule with isolated lines can be used. We elaborate on the method, substantiate the method by reporting results of this calibration procedure applied to multipass and single pass gas cells of lengths from $0.38 \mathrm{~m}$ to $21 \mathrm{~m}$, and compare this to other methods. The relative combined uncertainty of the path length results determined using the comparison method was found to be in the $\pm 0.4 \%$ range.
\end{abstract}

\section{Introduction}

Many absorption spectroscopy techniques such as tunable diode laser absorption spectroscopy (TDLAS), quantum cascade laser absorption spectroscopy (QCLAS), or Fourier transform infrared (FTIR) spectroscopy rely on the accurate knowledge of the optical path length of the absorption cell of the spectrometer wherein the gas sample interacts with the photons [1-6]. The determination of the optical path length of the gas cell prior to any quantitative spectroscopic analysis is indispensable in applications which aim at absolute measurements; in this case an incorrect path length of a gas cell would automatically lead to an incorrect result. If traceability should be an issue for the final spectroscopic analysis result, the determination of the optical path length also has to be traceably determined. The optical path length calibration (OPC), therefore, takes an important position in the characterization and validation of absolute absorption spectrometer setup.

There are several methods by which an OPC of a gas cell could be accomplished. Typically, in laser absorption spectroscopy, the same laser spectrometer setup is used to calibrate the path length of the cell by means of a gas mixture of previously known concentration as discussed in [7]. Assuming that all other parameters, both the spectroscopic and the gas condition ones, are exactly known, the spectroscopic signal of the spectrometer is taken to match the path length of the gas cell used so that the spectroscopic concentration result exactly reflects the reference concentration. This, however, is not independent of the application, that is, the concentration measurement.

The path length of a single pass cell can easily be determined using mechanical measurements. However, for single pass cell lengths in the few millimeters range, even mechanical measurements might be challenging to carry out. On the other hand, multipass gas cells, such as those of the Herriott et al. [8] or White [9] type, are used in most laboratories dealing with spectroscopic analysis in order to increase the spectrometer sensitivity. For those multipass gas cells, it can become even more challenging to determine the path length since multiple reflections are involved, and the effective optical length is difficult to assess. Mechanical measurements can, if at all, only yield some geometrical base length of which the actual optical path is often supposed to be an integer multiple. 


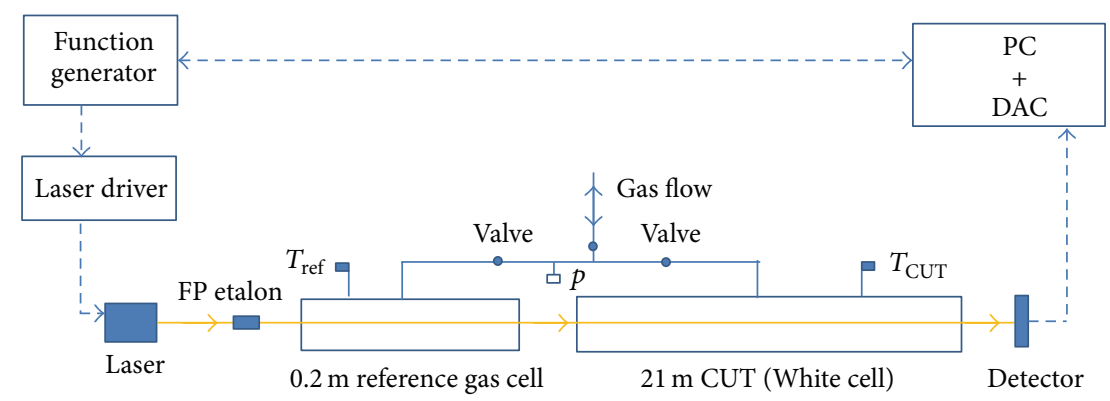

FIGURE 1: Schematic diagram of the setup used to calibrate the optical path length of a gas cell under test (CUT) with $L=21 \mathrm{~m}$ (nominally). The reference cell has a nominal optical path length of $0.2 \mathrm{~m}$. PD: photodiode. FP: Fabry-Perot etalon (removable).

Although the OPC is an essential part of a spectrometer characterization, its realization and interpretation still seem to manifest some issues and difficulties. Even in the gas metrology area, the OPC was identified as one of the major concerns in the outcome of a recent spectroscopic study [10].

In this paper, we promote an accurate easy to use method to calibrate the optical path length of a gas cell by means of laser spectroscopy. We show that the path length of a gas cell under test (CUT) can accurately be determined by a comparison to a reference cell of known path length. The quality of the presented OPC approach is superior to most simple mechanical measurements, approaches for multipass gas cells which count the number of spots on the cell mirrors, or to the use of gas standards. Traceability to the SI is possible with the OPC promoted here. We support the described implementation of this OPC method by presenting results on calibrating the optical path length of a multipass gas cell and a couple of single pass cells. The results are compared to mechanical path length measurements. In separate experiments, we validated each derived optical path length by measuring amount fractions of gravimetrically prepared gas mixtures. Finally, we present and discuss some recommendations for the proposed method.

\section{New OPC Method}

In laser absorption spectroscopy, the Beer Lambert law describes the relationship between the incident and the transmitted radiation through a gas cell containing a molecular gas sample:

$$
\Phi(\widetilde{\nu}, L)=\Phi_{0}(\widetilde{\nu}) \cdot \exp \left\{-S_{T} \cdot r_{\text {iso }} \cdot g\left(\widetilde{\nu}-\widetilde{v}_{0}\right) \cdot L \cdot n\right\} .
$$

The quantities $\Phi_{0}$ and $\Phi$ are the incident and the transmitted radiant powers; $S_{T}$ is the molecular transition line strength of the probed absorption line at gas temperature $T$ which is matched to the actually present isotopic abundance by means of $r_{\text {iso }}$, which is the ratio of the abundance in the sample compared to the value for which the line strength is reported. The function $g$ is the normalized line shape profile centered at $\widetilde{\nu}_{0}$ and $L$ the absorption path length. Using the ideal gas law, the number density $n$ of the absorbing species can be expressed in terms of the partial pressure $p_{\text {partial }}$ of the absorbing molecules and the gas temperature. The partial pressure $p_{\text {partial }}$ can be related to the total pressure $p_{\text {total }}$ using the amount of substance fraction of the absorbing species, $x_{\text {species }}=p_{\text {partial }} / p_{\text {total }}$. Making use of the area normalization of $g$, (1) can also be written as

$$
\begin{aligned}
x_{\text {species }} & =\frac{k_{\mathrm{B}} \cdot T}{S_{T} \cdot r_{\text {iso }} \cdot L \cdot p_{\text {total }}} \int_{-\infty}^{\infty} A(\widetilde{\nu}) d \widetilde{v} \\
& =\frac{k_{\mathrm{B}} \cdot T}{S_{T} \cdot r_{\text {iso }} \cdot L \cdot p_{\text {total }}} \cdot A_{\text {line }},
\end{aligned}
$$

where the quantity $A(\widetilde{\nu})=-\ln \left(\Phi(\widetilde{\nu}) / \Phi_{0}(\widetilde{\nu})\right)$ is the spectral absorbance (Naperian), in certain cases (see, e.g., http://goldbook.iupac.org/A00028.html) also called extinction, and $A_{\text {line }}$ is the spectrally integrated area of the absorption line. Using (2) the species amount fraction can be derived from spectroscopic measurements as pointed out in previous publications [12]. This relation however can also be exploited for the OPC proposed by the present work.

Figure 1 depicts the setup used for the path length calibration. It typically contains an infrared diode laser, a FabryPerot etalon, a photodiode, and a pressure and temperature sensor similar to the setup in [12]. The two cells in Figure 1 are connected to each other and the same pressure sensor is used to determine the total gas pressure.

In Figure 1 the reference gas cell has an optical path length of $L_{\text {ref }}=0.20 \mathrm{~m}$ and the cell under test (CUT) in this case has a nominal optical path length of $21 \mathrm{~m}$. We used a serial arrangement in the experiment in order to keep with a single detector as opposed to a parallel arrangement where two detectors would have been required.

If the CUT is evacuated and the reference cell is filled with a gas sample, its spectrometric amount fraction $x_{\text {species }}$ can be expressed by (2) where $L$ is substituted by $L_{\text {ref }}, p_{\text {total }}$ by $p_{\text {ref }}$, and $T$ by $T_{\text {ref }}$, respectively. On the other hand, if the reference cell is evacuated and the gas cell under test is filled with the same gas sample, its spectrometric amount fraction $x_{\text {species }}$ is given by (2) where $L$ means the length to be calibrated and $p_{\text {total }}$ and $T$ are substituted by $p_{\text {CUT }}$ and $T_{\text {CUT }}$, respectively.

Since the same gas was used in the reference cell and the CUT and the same transition was probed, both sides of (2) can be replaced to then read

$$
\frac{k_{\mathrm{B}} \cdot T_{\text {ref }}}{S_{T_{\text {ref }}} \cdot r_{\text {iso }} \cdot L_{\text {ref }} \cdot p_{\text {ref }}} \cdot A_{\text {ref }}=\frac{k_{\mathrm{B}} \cdot T_{\mathrm{CUT}}}{S_{T_{\mathrm{CUT}}} \cdot r_{\text {iso }} \cdot L \cdot p_{\mathrm{CUT}}} \cdot A_{\mathrm{CUT}},
$$




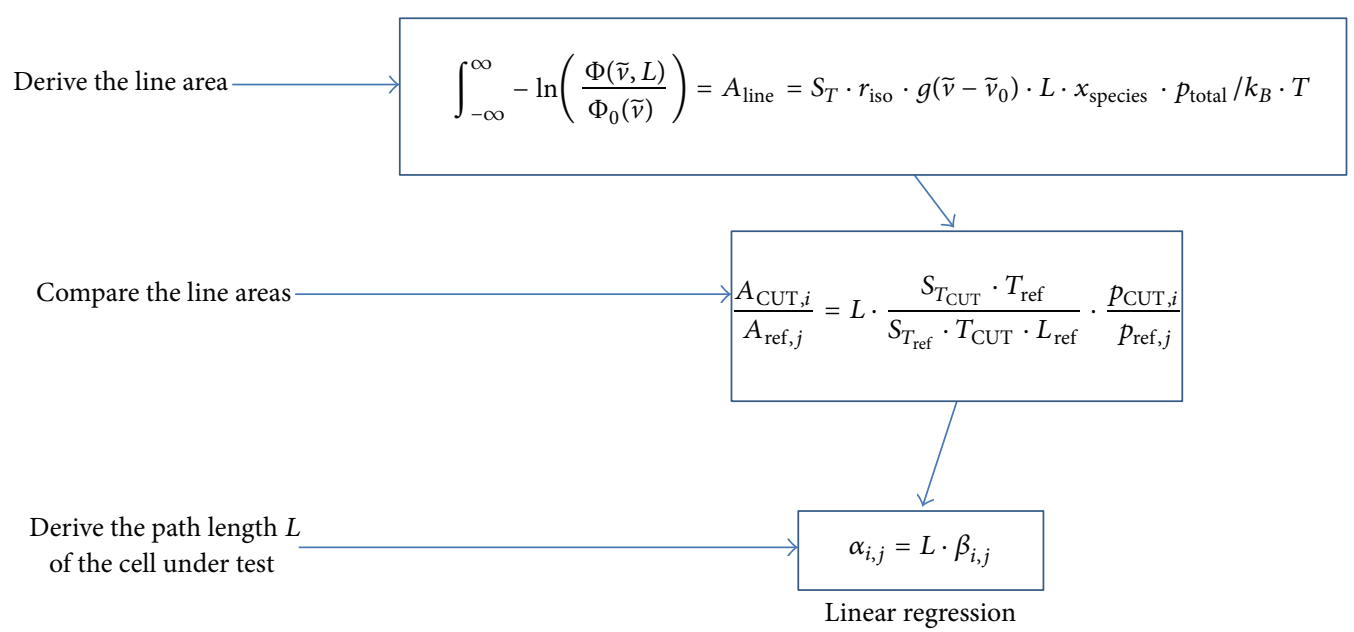

FIGURE 2: Scheme of the OPC procedure.

where $A_{\text {ref }}$ and $A_{\text {CUT }}$ denote the line areas of the probed spectral absorption feature in the case of the reference and the gas cell under test, respectively. As presented in (3), the exact knowledge of the amount fraction of the gas mixture is not necessary in the calibration of length $L$. Rearrangement of (3) leads to the model equation of the proposed OPC:

$$
\alpha_{i, j}=L \cdot \beta_{i, j}
$$

where $\alpha_{i, j}=A_{\mathrm{CUT}, j} / A_{\text {ref }, i}$ and $\beta_{i, j}=\left(S_{T_{\mathrm{CUT}}} \cdot T_{\text {ref }} / S_{T_{\mathrm{ref}}}\right.$. $\left.T_{\text {CUT }} \cdot L_{\text {ref }}\right) \cdot\left(p_{\text {CUT, } j} / p_{\text {ref }, i}\right)$, and to the premise that the same spectral feature, that is, the same molecular absorption line of the strength $S$, was probed in both cells. The indices $i$ and $j$ indicate that multiple cell measurements could be repeated at variable gas pressures in both or one of the cells, thus adding some redundancy to the OPC. The gas temperatures $T_{\text {ref }}$ and $T_{\text {CUT }}$ at the different pressures were recorded. This redundancy of the OPC can then be used to fit a linear line model to the data points $\left(\alpha_{i, j} ; \beta_{i, j}\right)$ by applying generalized linear regression (GLR) according to (4). From this linear model the slope is identified to deliver the path length $L_{\text {OPC }}$.

In the case that for both cell measurements the same total gas pressure $p_{i}$ at the same gas temperature was realized, (4) would reduce to

$$
\alpha_{i=j} \cdot p_{i}=L \cdot \frac{p_{i}}{L_{\mathrm{ref}}}
$$

and the linear model would have to consider the regression on data points $\left(\alpha_{i=j} \cdot p_{i} ; p_{i} / L_{\text {ref }}\right)$. For the simplified condition expressed in (5), it can be observed that the line area ratio of the two cells is just equal to the length ratio. The described OPC procedure would yield a result $L_{\mathrm{OPC}}$ which is traceable to the SI if all the input quantities $A, S_{T}, T$, and $p$ used in (4) were traceable.

It should be noted here that, if both cell measurements would have been done at different gas temperatures instead, the line strengths would not be cancelled out and were to be matched to the temperatures of the reference and the test cell measurements, respectively, according to

$$
\begin{aligned}
S_{T}= & S_{0} \cdot\left(\frac{Q_{T_{0}}}{Q_{T}}\right) \cdot \exp \left\{-h \cdot c \cdot \frac{E^{\prime \prime}}{k_{\mathrm{B}}} \cdot\left(\frac{1}{T}-\frac{1}{T_{0}}\right)\right\} \\
& \cdot \frac{\left[1-\exp \left\{-h c \cdot\left(\widetilde{v}_{0} / k_{\mathrm{B}} T\right)\right\}\right]}{\left[1-\exp \left\{-h c \cdot\left(\widetilde{v}_{0} / k_{\mathrm{B}} T_{0}\right)\right\}\right]}
\end{aligned}
$$

where $Q_{T_{0}}$ and $Q_{T}$ are the internal partition functions of the probed molecule at the reference temperature $T_{0}=296 \mathrm{~K}$ and $T$, respectively. $E^{\prime \prime}$ is the ground state energy of the probed transition, $\widetilde{v}_{0}$ is the spectral transition wave number, $h$ is the Planck constant, and $c$ is the speed of light in vacuum. Because the line strength $S_{0}$ at $T_{0}$ is the same for both cells, only the temperature dependence of the line strength would have to be considered in the determination of $L_{\mathrm{OPC}}$.

Figure 2 depicts a schematic diagram of the OPC method. The general idea of the presented OPC procedure is based on a comparison of spectral features measured on the very same gas sample.

The feasibility of the method depends on the quality of the two spectra measured in the two cells, that is, on $A_{\text {ref }}, A_{\text {CUT }}$, and their associated uncertainties $u\left(A_{\text {ref }}\right)$ and $u\left(A_{\mathrm{CUT}}\right)$, respectively. Probing the same spectral line in both cells, a large amplitude difference of the two spectral features due to the different path lengths can be compensated for by means of different gas pressures in both cells. Thus, the absorption line in both cell spectra can be balanced so that the peak absorbance is still in the linear regime for a large $L_{\text {OPC }}$ and well above the noise level for a much smaller $L_{\text {ref. }}$.

The usability of the method to calibrate a path length $L$ depends on the availability of a suitable reference cell, characterized by $L_{\text {ref }}$ and $u\left(L_{\text {ref }}\right)$, and can be expressed by the dynamic range of this OPC method defined as the maximum length ratio $r_{L, \max }=L_{\text {max }} / L_{\text {ref }}$ for which the inferred length uncertainty is smaller than the value $u_{\max }\left(L_{\mathrm{OPC}}\right)$ tolerable for a given experimental task. A certain reference cell can be used to calibrate a number of path lengths up to $L_{\max }$, if its uncertainty applies to $u\left(L_{\max }\right) \leq u_{\max }(L)$. The uncertainty $u\left(L_{\mathrm{OPC}}\right)$ of the calibrated optical path length $L_{\mathrm{OPC}}$ is a 


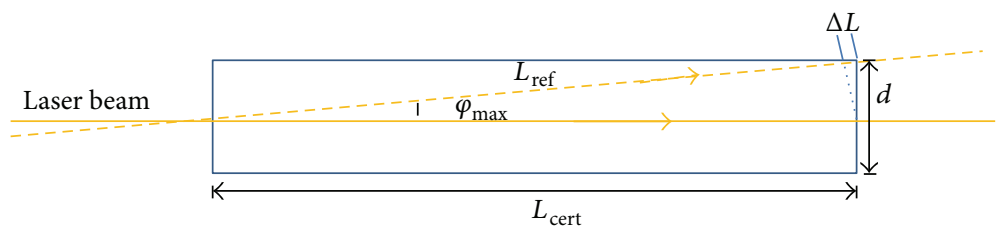

FIgURE 3: Schematic of a possible laser light path deviation for the reference gas cell (worst case scenario).

function of the uncertainties $u\left(L_{\text {ref }}\right), u\left(A_{\text {ref }}\right)$, and $u\left(A_{\text {CUT }}\right)$ and of those of the pressure and temperature measurements $u\left(p_{\text {ref }}\right), u\left(p_{\text {CUT }}\right), u\left(T_{\text {ref }}\right)$, and $u\left(T_{\text {CUT }}\right)$ if pressures and temperatures were different in both cells.

\section{Experimental OPC Results}

3.1. Calibration of the Path length of a Multipass Gas Cell (White Cell). The setup sketched in Figure 1 was used to calibrate the path length of a White cell of a nominal path length of $L=21 \mathrm{~m}$ by means of a reference gas cell of an optical path length of $L_{\text {ref }}=(0.2003815 \pm 0.000062) \mathrm{m}$. This reference cell path length was modeled as $L_{\text {ref }}=L_{\text {cert }}+$ $\delta L$, where $L_{\text {cert }}=0.2003815 \mathrm{~m}$ is the certified geometrical cell length and $\delta L$ is a possible correction due to some possible deviation $\varphi$ of the actual laser beam path from the optical axis. As sketched in Figure 3, this correction can be expressed as $\delta L=L_{\text {cert }} \times(1 / \cos \varphi-1)$ with $|\varphi| \leq\left|\varphi_{\max }\right|$, and $2 \times \tan \varphi_{\max }=d / L_{\text {cert }}$ when $d=0.01 \mathrm{~m}$ is the free optical aperture of the cell. The combined uncertainty of the reference gas cell optical path length $u\left(L_{\text {ref }}\right)$ was estimated from the geometrical length uncertainty $u\left(L_{\text {cert }}\right)=2.5 \cdot 10^{-7} \mathrm{~m}(k=1)$ taken from the certificate of the geometrical reference length measured by the Coordinate Metrology Department of PTB employing a coordinate measurement machine (CMM, Type Leitz PMM866) (http:// www.ptb.de/cms/en/fachabteilungen/abt5/fb-53/ag-535.html) and an uncertainty contribution $u(\delta L)$. While our estimate for the laser beam path was taken to be $\varphi=0$, we calculated a conservative scenario setting the uncertainty to be $u(\delta L)=\delta L\left(\varphi=\varphi_{\max }\right)=0.062 \mathrm{~mm}$, by overestimating a rectangular probability distribution that $\varphi$ is within the interval $\left[-\varphi_{\max } ; \varphi_{\max }\right]$. Finally the reference cell path length uncertainty was estimated from $u^{2}\left(L_{\text {ref }}\right)=u^{2}\left(L_{\text {cert }}\right)+u^{2}(\delta L)$.

For this OPC, a distributed feedback (DFB) diode laser emitting at $2 \mu \mathrm{m}$ was used. In order to perform the measurements, the wavelength of the DFB laser was swept by triangular modulation of the laser current at a frequency of $138.9 \mathrm{~Hz}$. For signal detection, a photodiode was employed from which the signal was digitized by a 16-bit data acquisition card (DAC) at a sampling rate of $1.2 \mathrm{MS} / \mathrm{s}$.

In order to calibrate the path length $L$ of the multipass gas cell, a gas mixture of $\mathrm{CO}_{2}$ in $\mathrm{N}_{2}$ was used. The $\mathrm{R}(12)$ line of $\mathrm{CO}_{2}$ at $4987.31 \mathrm{~cm}^{-1}$ was probed. As mentioned before, information on the exact amount fraction of the target species in the gas mixture is not required for the OPC data processing.

For the OPC, both cells were evacuated and background measurements showed no $\mathrm{CO}_{2}$ absorption, for example, from residual gas in the cell or outside the cell pathlength. The baseline noise level was determined to be less than

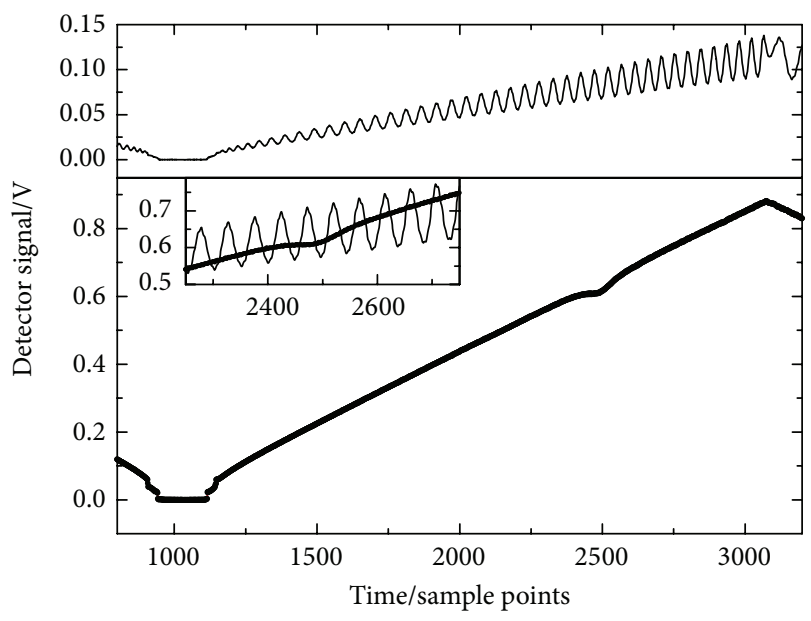

FIGURE 4: Detector signals with an etalon (top) in the beam path, with gas in the cell (bottom), and with both of them (inset), as a function of digital sample points. Data are on the reference cell containing $20 \mathrm{mmol} / \mathrm{mol} \mathrm{CO} 2$ in nitrogen gas mixture at $p_{\text {ref }}=$ $(201 \pm 0.2) \times 10^{2} \mathrm{~Pa}$ (bottom).

$1 \times 10^{-3}$ based on a $1-\sigma$ standard deviation of the residual of the absorption line fit. Then the reference cell was filled with the $\mathrm{CO}_{2}$-containing sample gas mixture. Spectroscopic measurements with the reference cell were carried out for different values of the total gas pressure $p_{\text {ref }, i}$ inside the reference cell according to (4), determining the respective line areas $A_{\text {ref,i }}$ for each pressure by means of fitting a single Voigt profile to each nonaveraged absorption profile. In the fitting process the Doppler-width was computed using the measured gas temperature $T$, while the Lorentzian width and the line area were fitted as well as the line position. Secondly, whereas the reference cell was evacuated again, the multipass cell under test was filled with the same gas mixture and measurements were performed for different gas pressures $p_{\mathrm{CUT}, j}$ according to (4), to determine the respective line areas $A_{\mathrm{CUT}, j}$ from the multipass cell's absorbance spectra. The gas pressure and the gas temperature were each measured with a pressure sensor (MKS Baratron) and a PT100 temperature sensor, respectively. After each change of the gas pressure in the cells, data were just monitored for a period of 10 minutes to accommodate thermodynamic equilibration of the gas sample prior to real spectra recording. Within the recording duration of all spectra, the stability in terms of the standard deviation of the line area, the temperature and the pressure of the gas sample was typically within $\pm 0.25 \%, \pm 0.07 \%$, and $\pm 0.14 \%$, respectively.

Figure 4 depicts typical data measured on a gas sample in the reference cell (nominally $20 \mathrm{mmol} / \mathrm{mol} \mathrm{CO}_{2}$ ) at a total 


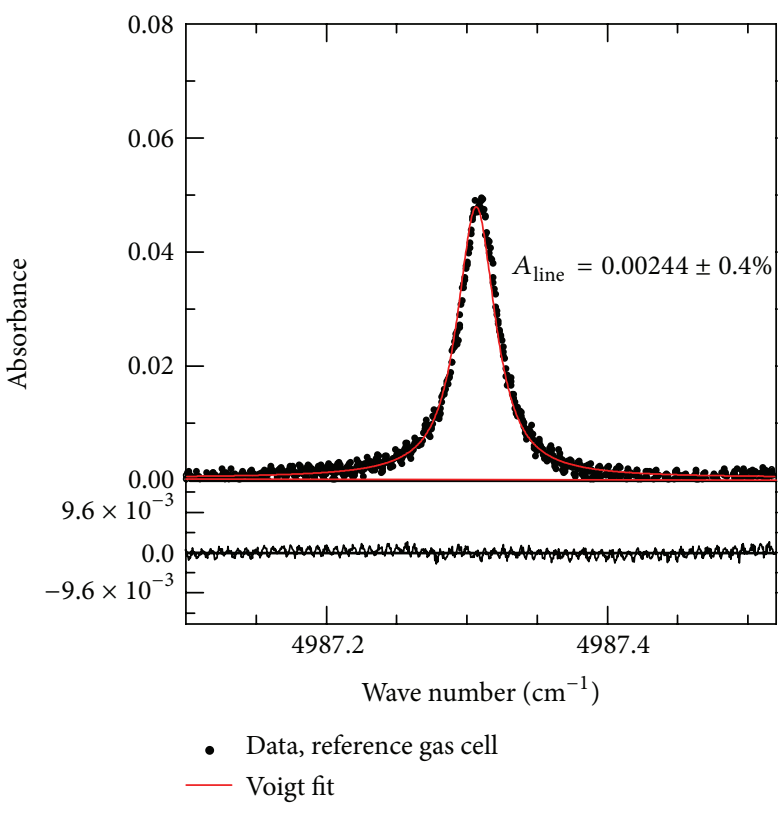

(a)

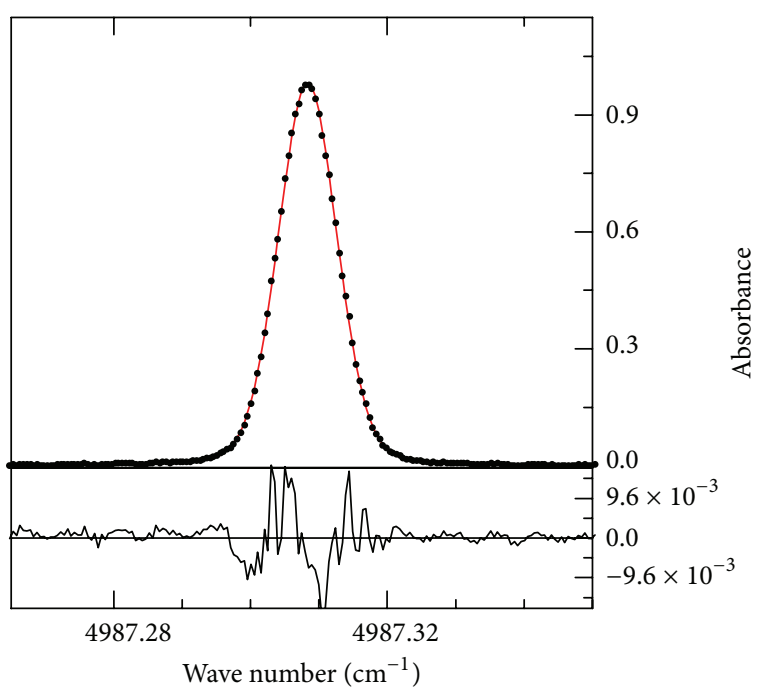

- Data, CUT

- Voigt fit

FIgure 5: $\mathrm{CO}_{2}$ line profile in the $20 \mathrm{~cm}$ reference cell at $185 \times 10^{2} \mathrm{~Pa}$ (a) and the multipass gas cell (nominal length $21 \mathrm{~m}$ ) at $6 \times 10^{2} \mathrm{~Pa}(\mathrm{~b})$. The optical path length of the multipass cell was to be calibrated.

pressure of $p_{\text {ref }}=(201 \pm 0.2) \times 10^{2} \mathrm{~Pa}$. The detector signal in the top panel of Figure 4 is the signal measured when a removable Fabry-Perot etalon is placed inside the beam path to the detector (cf. Figure 1) when both cells were evacuated. The etalon signal is used to establish a linear wave number axis by means of the sweep rate derived from the fringe separation as presented in [12-16]. The detector signal in the bottom panel of Figure 4, measured when the Fabry-Perot etalon was removed, shows the used absorption feature of $\mathrm{CO}_{2}$.

In Figure 5, line profiles $A(\widetilde{\nu})$ of the reference cell (Figure 5(a)) and the multipass cell under test (Figure 5(b)), respectively, are displayed. These absorbance data were derived from the measured signal in Figure 4 according to $A(\widetilde{\mathcal{\nu}})=-\ln \left(\Phi(\widetilde{\mathcal{\nu}}) / \Phi_{0}(\widetilde{\mathcal{\nu}})\right)$, where $\Phi_{0}(\widetilde{\mathcal{\nu}})$ was derived by fitting a polynomial baseline to the measured signal [13-17] for the reference cell and for the CUT accordingly.

Voigt profiles were subsequently fitted to the absorbance data in Figure 5 in order to derive values for the respective line areas for the given gas pressures, that is, $A_{\text {ref, } j}$ and $A_{\mathrm{CUT}, i}$, respectively. In this fitting process the Doppler-width was computed according to the measured gas temperature $T$, while the Lorentzian width and the line area as well as the exact line position were fitted. As plotted in the example spectra in Figure 5 the standard deviation of the residuals of the $0.2 \mathrm{~m}$ reference and the $21 \mathrm{~m}$ multipass gas cells was $1 \times$ $10^{-3}$ and $2 \times 10^{-3}$, resulting in signal-to-noise ratios of 50 and 494, respectively. Unlike the improved residual structure for the $20 \mathrm{~cm}$ gas cell at the lowest pressure of $185 \times 10^{2} \mathrm{~Pa}$ (see, e.g., Figure 5), the residual structure of the multipass gas cell has a typical " $w$ " structure indicating the presence of collisional narrowing at the lowest gas pressure of $6 \times 10^{2} \mathrm{~Pa}$.
Such oscillating structure has been shown to have a marginal effect on the line area [18]. Here, for the multipass gas cell, the residual structure area is $0.004 \%$ of the line area, which we consider to be negligible. The residual structure area was found to be $<0.004 \%$ for all other measurements presented in this paper. Hence, the " $\mathrm{w}-$ " shaped residual structure of the multipass gas cell has an insignificant effect on the line area. The derived line areas and the other input parameters were used to derive the values of $\alpha_{i, j}$ and $\beta_{i, j}$ and the line strength was matched to each of the measured gas temperatures $T_{\text {ref, } j}$ and $T_{\mathrm{CUT}, i}$ applying (6).

Figure 6(a) depicts a plot of $\alpha_{i, j}$ as a function of $\beta_{i, j}$. According to (4), the path length of the multipass gas cell $L_{\mathrm{OPC}}$ was determined from the slope parameter of the generalized linear regression (GLR) applied to the data points $\left(\alpha_{i, j}, \beta_{i, j}\right)$. In compliance with the GUM [19] the GLR was performed by means of the BLeast software [20] which is well known from ISO 6143 [21]. Input uncertainties for this GLR were taken as reported in Table 2. The path length of the CUT resulting from the data in Figure 6(a) was evaluated to be $(21.87 \pm 0.04) \mathrm{m}$. A combined uncertainty of $\pm 0.04 \mathrm{~m}$ $( \pm 0.20 \%$ relative $)$ was estimated from the approach. It should be noted that both the uncertainties of $\alpha_{i, j}$ and $\beta_{i, j}$ were taken into account applying the GLR.

3.2. Calibration of the Path Length of a Single Pass Gas Cell. Another OPC was carried out in a similar manner, this time calibrating the path length of a single pass gas cell of a nominal length of $82 \mathrm{~cm}$. For this OPC, however, a different spectrometer setting and a different gas mixture of $10 \mathrm{mmol} / \mathrm{mol} \mathrm{CH}_{4}$ in synthetic air were utilized to show the flexibility of the OPC method. In the spectrometer, a DFB 
TABLE 1: Comparison of path lengths derived from the presented OPC $\left(L_{\mathrm{OPC}}\right)$, from mechanical measurements $\left(L_{\text {mech }}\right)$, and from gas standardbased retrieval $\left(L_{\text {gas }}\right)$, respectively. Given are normalized errors $E_{n}$ referring to $L_{\mathrm{OPC}}$.

\begin{tabular}{lcccccccc}
\hline Cell & Cell type & $L_{\mathrm{OPC}} / \mathrm{m}$ & $L_{\text {mech }} / \mathrm{m}$ & $\left|E_{n}(\mathrm{mech})\right|$ & $L_{\text {gas }} / \mathrm{m}$ & $\mid E_{n}($ gas $) \mid$ & Species $^{\mathrm{a}}$ & $\lambda / \mu \mathrm{m}$ \\
\hline A & MP $^{*}$ & $21.87 \pm 0.040$ & - & - & $21.26 \pm 0.31$ & 0.9 & $\mathrm{CO}_{2}$ & 2.0 \\
B & SP, HP $^{*}$ & $0.819 \pm 0.0029$ & $0.822 \pm 0.0010$ & 0.5 & $0.805 \pm 0.041$ & 0.2 & $\mathrm{CH}_{4}$ & 1.65 \\
C & SP, BW $^{*}$ & $0.773 \pm 0.0020$ & $0.774 \pm 0.0010$ & 0.2 & $0.773 \pm 0.038$ & 0.0 & $\mathrm{CH}_{4}$ & 1.65 \\
C & SP, BW $^{*}$ & $0.773 \pm 0.0020$ & $0.774 \pm 0.0010$ & 0.2 & $0.774 \pm 0.012$ & 0.04 & $\mathrm{CO}_{2}$ & 2.71 \\
D & SP, HT $^{*}$ & $0.380 \pm 0.0012$ & $0.380 \pm 0.0020$ & 0.0 & $0.380 \pm 0.019$ & 0.0 & $\mathrm{CH}_{4}$ & 1.65 \\
\hline
\end{tabular}

${ }^{\mathrm{a}}$ Gas standards probed at wavelengths specified in $\lambda$ column.

${ }^{*}$ Cell characteristics: MP multipass White type, SP single pass cell, HP high pressure cell, BW Brewster windows-equipped, and HT high temperature cell.

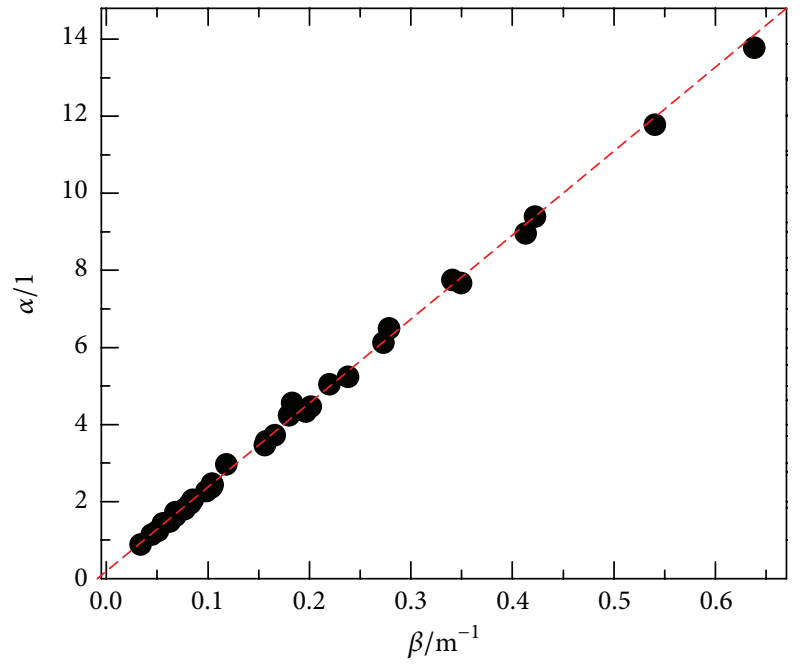

- Measured data

--- GLR

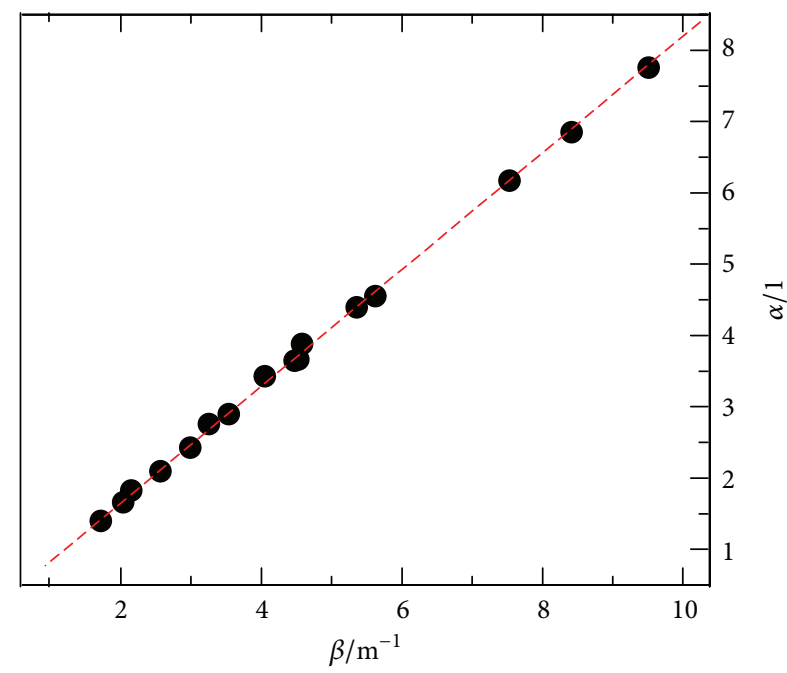

- Measured data

--- GLR

(a) $21 \mathrm{~m}$ gas cell

(b) $82 \mathrm{~cm}$ gas cell

FIGURE 6: OPC data $\alpha_{i j}$ as a function of $\beta_{i j}$ processed to retrieve the optical path lengths of the CUTs A (a) and B (b) of $L_{\mathrm{OPC}}=(21.89 \pm$ $0.08) \mathrm{m}$ and $L_{\mathrm{OPC}}=(0.819 \pm 0.0029) \mathrm{m}$, respectively. For (a) a mixture of about $20 \mathrm{mmol} / \mathrm{mol} \mathrm{CO} 2$ in nitrogen was used, whereas for (b) a mixture of $10 \mathrm{mmol} / \mathrm{mol} \mathrm{CH}_{4}$ in nitrogen was probed for the OPC.

TABLE 2: Relative standard uncertainties of the most impacting input quantities.

\begin{tabular}{lcccc}
\hline Quantity & $p$ & $T$ & $A_{\text {line }}$ & $L_{\text {ref }}$ \\
\hline$u_{\text {rel }} / \%$ & 0.16 & 0.08 & 0.40 & 0.03 \\
\hline
\end{tabular}

diode laser emitting at $1.65 \mu \mathrm{m}$ was used and a $\mathrm{CH}_{4}$ line at $6046.96 \mathrm{~cm}^{-1}$ was probed modulating the laser current again at $138.9 \mathrm{~Hz}$. Data acquisition now took place at a sampling rate of $600 \mathrm{kS} / \mathrm{s}$ with the same DAC. The fitting procedure for the $\mathrm{CH}_{4}$ absorbance data was performed in a manner similar to the data presented in Figure 5. Here, it should be noted once more that the proposed OPC method is independent of the exact amount fraction of the gas mixture, the probed molecule, and the IR light source.

Figure 6(b) depicts $\left(\alpha_{i, j}, \beta_{i, j}\right)$ data, similar to Figure 6(a), which were processed by GLR to determine the path length of the $82 \mathrm{~cm}$ single pass gas cell. The slope of the linear model fitted to the data resulted in a path length of the single pass cell of $L=81.90 \mathrm{~cm}$. Again, the BLeast software was used to perform the GLR. The combined uncertainty of the path length was estimated to be $u\left(L_{\text {OPC }}\right)= \pm 0.29 \mathrm{~cm}( \pm 0.35 \%$ relative).

\section{Discussions}

The OPC method described was employed to calibrate a number of gas cells for their use in different spectroscopic applications. For the present paper, results on four different cells are summarized in Table 1 . Based on these results the OPC method was also brought forward as a standard approach (SA) for OPC in our lab and is about to be established as SA in the EUMETRISPEC joint research project [22].

Table 1 summarizes the OPC results in terms of the derived $L_{\mathrm{OPC}}$ and $u\left(L_{\mathrm{OPC}}\right)$ and compares them to respective results on simple mechanical measurements reported as $L_{\text {mech }}$ and $u\left(L_{\text {mech }}\right)$, respectively, where applicable, and to results $L_{\text {gas }}$ and $u\left(L_{\text {gas }}\right)$ which are based on the use of gas standards of amount fractions $x$ according to the rearrangement 
of (2) to yield $L$ as a result. The mechanical measurements were performed using a meter rule and a caliper (resolution: $0.001 \mathrm{~m}$ ) calibrated against length standards.

The relative combined uncertainties $u\left(L_{\text {OPC }}\right)$ of the OPC path length results in Table 1 are in the $0.2-0.4 \%$ range. Table 2 holds the standard uncertainties $(k=1)$ of the most significant input quantities in (3) and (4) used to determine $u\left(L_{\mathrm{OPC}}\right)$.

As shown in Table 2, the input quantity with the highest relative uncertainty is the line area. As such, it provides the most significant uncertainty contribution to the combined uncertainty of the path length $L_{\mathrm{OPC}}$. The standard uncertainty of $A_{\text {line }}$ was estimated from fitting a Voigt profile to the measured absorbance data. For this, the Origin fitting error output [23] was used, derived on the most noisy and least signal-to-noise spectra as a conservative estimate (see, e.g., Figure 5(a)). It could be noted here that for the OPC method we used the same line profile, the same spectral window, and the same wave number axis to model the spectra from the two cells in a similar manner, and since we were dealing with the ratio of two line areas, the uncertainty contribution of the wave number axis to the line area uncertainty was eliminated from our analysis. Any uncertainty contribution to the line area as a result of the residual structure was neglected, since the area from the residuals of the multipass gas cell in Figure 5 was negligible (max. $0.004 \%$ of the line area) for all measurements. The uncertainties of the other input quantities, that is, $p, T$, and $L_{\text {ref }}$, were propagated from respective calibration certificates. The traceability to the SI of the input parameters $p$ and $T$ was reached via respective standards.

The relative combined uncertainties of $L_{\text {mech }}$ in Table 1 are in the $\pm 0.53 \%$ range. The combined uncertainties of $L_{\text {mech }}$ were estimated using the resolution of the caliper. The quantity $E_{n}(\mathrm{mech})$ is the normalized error of the mechanical length result expressed as

$$
E_{n}=\frac{D}{U(D)},
$$

where $D=L-L_{\text {mech }}$ and $U(D)=2 \times u(D)=2 \times$ $\sqrt{\left(u^{2}(L)+u^{2}\left(L_{\text {mech }}\right)\right)}$. The $E_{n}($ mech $)$ are all less than 1 , indicating that the results of $L_{\mathrm{OPC}}$ from the OPC approach for the different gas cells agree with the respective values of $L_{\text {mech }}$. This agreement of $L_{\mathrm{OPC}}$ and $L_{\text {mech }}$ validates the presented OPC method. Although it is much more complicated than mechanical measurements, the OPC method presented in this paper was still being applicable in cases where path lengths cannot be determined by mechanical measurements, for example, in multipass gas cells and very short length single pass gas cells. For those cells the OPC method is probably the best candidate to be utilized for optical path length determination.

Table 1 also shows an agreement between $L_{\mathrm{OPC}}$ from the OPC and $L_{\text {gas }}$ as documented by the values of $E_{n}(x)$ being all less than 1 . However, the uncertainties of $L_{\text {gas }}$ in Table 1 are about 13 times larger (up to 5\%) than the uncertainties of $L_{\text {OPC }}$ which are found to be in the $\pm 0.4 \%$ range instead. Also,
TABLE 3: Uncertainty budget of $L_{\text {gas }}$ for gas cell B $(82 \mathrm{~cm})$ derived by means of (2) with $\mathrm{CH}_{4}$ being the probed sensor molecule. The index column is the relative contribution of the properties variance to the variance of $L_{\text {gas }}[11]$.

\begin{tabular}{|c|c|c|c|}
\hline $\begin{array}{l}\text { Input } \\
\text { quantity }\end{array}$ & Value & $\begin{array}{c}\text { Relative } \\
\text { uncertainty/\% }\end{array}$ & Index/\% \\
\hline$p$ & $244 \mathrm{hPa}$ & 0.16 & 0.0 \\
\hline$T$ & $294.8 \mathrm{~K}$ & 0.08 & 0.1 \\
\hline$A_{\text {line }}$ & $0.00402 \mathrm{~cm}^{-1}$ & 0.4 & 0.6 \\
\hline$x_{\text {species }}$ & $0.01 \mathrm{~mol} / \mathrm{mol}$ & 0.99 & 3.7 \\
\hline$S_{0}$ & $\begin{array}{c}8.289 \times 10^{-22} \\
\mathrm{~cm}^{-1} /\left(\mathrm{cm}^{-2} \cdot \mathrm{molec}\right)\end{array}$ & 5 & 95.4 \\
\hline$L_{\text {gas }}$ & $0.805 \mathrm{~m}$ & 5 & \\
\hline
\end{tabular}

the uncertainties of $L_{\mathrm{gas}}$ in Table 1 are 9 times larger than the uncertainties of $L_{\text {mech }}$.

These large values of the uncertainties of $L_{\text {gas }}$ are a result of the uncertainties of the used line strength figures. In Table 3, an example uncertainty budget for $L_{\text {gas }}$ is presented, derived using the GUM Workbench [11] which shows the impact of the line strength uncertainty on the combined uncertainty of $L_{\text {gas }}$. As can be seen from Table 3, the index value [11] (relative contribution of the uncertainties of an input quantity to the combined uncertainty of $L_{\text {gas }}$ ) of the line strength is $99.1 \%$, indicating that the line strength's uncertainty provides the most significant contribution to $u\left(L_{\text {gas }}\right)$. For spectroscopic data evaluation in applications, such as environmental monitoring, where uncertainties in the subpercentage range are often requested, these large $u\left(L_{\text {gas }}\right)$ discourage the use of $L_{\text {gas }}$.

Regarding line strengths, the values and their uncertainties are usually measured or taken from spectroscopic data bases such as HITRAN [24] or GEISA [25]. However, traceable line strength values and GUM-compliant uncertainty figures are very rarely reported in the literature or data bases [26]. Hence, it is very likely that path lengths derived as $L_{\text {gas }}$ could be biased. The line strength values used for the evaluation of $L_{\text {gas }}$ in Table 3 were taken from the HITRAN [24] data base, except for $L_{\text {gas }}$ for the multipass gas cell (A) where the line strength was measured by PTB $[27,28]$. The relative standard uncertainties of the HITRAN line strengths used here are in the $2-5 \%$ range [24]. On the contrary, while omitting the line strength value, smaller uncertainties and traceability to the SI could be reached by means of the OPC method presented here. As presented and in addition, the following advantages solidify the OPC method:

(i) the method does not depend on a specific analyte; any infrared active molecule with a sufficiently isolated line can be used for the OPC method (depending on the light source);

(ii) knowledge of the exact amount fraction of the probed species in the gas sample is not necessary; since $x_{\text {species }}$ is not used in (4), its uncertainty does not contribute to the uncertainty of $L_{\mathrm{OPC}}$; 
(iii) OPC as presented here in general does not depend on any line strength parameter $S_{0}$, so that its uncertainty, for example, taken from existing data bases, does not contribute to the uncertainty of $L_{\mathrm{OPC}}$;

(iv) large amplitude differences in the absorption signal due to large length ratios $r_{L}$ can be compensated by appropriate pressure differences between the two cell measurements;

(v) the quantities contributing to the path length uncertainty $u\left(L_{\mathrm{OPC}}\right)$ can be minimized if pressure and temperature of both gas cells are always the same in the respective spectroscopic measurements, thus reducing the path length uncertainty $u\left(L_{\mathrm{OPC}}\right)$;

(vi) the method is independent of the actual application; that is, finally, the spectrometer's path length figure is independent of any concentration retrieval performed with the spectrometer.

If needed and if traceable line strengths data with low uncertainties were available, the OPC procedure could be made even more flexible on the other side. For a given reference cell of length $L_{\text {ref }}$ the dynamic length range $r_{L, \max }$ that can be covered can be remarkably exceeded if one probes two different absorption lines, giving rise to the modified expression $\beta_{i, j}=\left(r_{\text {iso,CUT }} \cdot S_{\text {CUT, } T_{\text {CUT }}} / r_{\text {iso,ref }} \cdot S_{\text {ref, } T_{\text {ref }}} \cdot\right.$ $\left.L_{\text {ref }}\right) \cdot\left(T_{\text {ref }} / T_{\text {CUT }}\right) \cdot\left(p_{\text {CUT }, i} / p_{\text {ref }, j}\right)$, thus considering different individual line strengths $S_{\mathrm{CUT}, T_{\mathrm{CUT}}}$ and $S_{\text {ref, } T_{\text {ref }}}$ for two different lines of the same gas probed in the two cells. Using different absorption lines, for example, from different bands, branches, and/or species isotopologues, with gradually different line strengths, the dynamic range $r_{L, \max }$ can be varied in principle by several orders of magnitude. Each of these line strengths have to be matched to the isotopic abundance actually present in the gas sample by means of $r_{\text {iso }}$. This idea, however, has the drawback of introducing additional uncertainty contributions regarding the line strengths $S_{0 \text {,ref }}$ and $S_{0, \text { CUT }}$ and the isotopic abundance factors $r_{\text {iso,ref }}$ and $r_{\text {iso, CUT }}$, respectively. They all individually contribute and thus definitely enlarge $u\left(L_{\mathrm{OPC}}\right)$ compared to the initial concept of probing the very same transition.

\section{Conclusion}

We presented a spectroscopic method to measure traceable optical path lengths of an absorption cell. We showed that the optical path length of a gas cell can be accurately calibrated comparing absorption spectra using the OPC method. For this paper, the OPC method was combined with tunable diode laser absorption spectroscopy. The relative uncertainties of our OPC results were in the range of 0.2 to $0.4 \%$. This OPC method leads to a significant improvement in cases where mechanical path length determination is difficult for gas cells because of geometrical or operational reasons. It is also advantageous with respect to the use of gas standards for optical path length measurements. This is because the latter directly depends on the exact knowledge of molecular line strengths, whose uncertainties are sometimes comparably large. Traceability to the SI is also lacking in most cases. The dynamic range and the flexibility of this OPC method are related to the availability of a reference cell with path length accuracy that exceeds the desired path length accuracy of the cell under test. The OPC method is not limited to TDLAS; other absorption spectroscopic techniques such as QCLAS or FTIR could be used in a similar manner.

\section{Conflict of Interests}

The authors declare that there is no conflict of interests regarding the publication of this paper.

\section{Acknowledgments}

Parts of this work received funding from EMRP projects. The authors acknowledge fruitful collaboration within the EMRP projects GAS (ENG01), MACPoll (ENV01), METEOMET (ENV07), and EUMETRISPEC (ENV06). The EMRP is jointly funded by the EMRP participating countries within EURAMET and the European Union. The authors are grateful to E. Gettkandt and K. Wendt for the calibration of the reference cell under Reference no. 5.3-2013-014.

\section{References}

[1] P. Ortwein, W. Woiwode, S. Fleck et al., "Absolute diode laser-based in situ detection of $\mathrm{HCl}$ in gasification processes," Experiments in Fluids, vol. 49, no. 4, pp. 961-968, 2010.

[2] G. Wysocki, M. McCurdy, S. So et al., "Pulsed quantum-cascade laser-based sensor for trace-gas detection of carbonyl sulfide," Applied Optics, vol. 43, no. 32, pp. 6040-6046, 2004.

[3] J. A. Nwaboh, O. Werhahn, and D. Schiel, "Measurement of $\mathrm{CO}$ amount fractions using a pulsed quantum-cascade laser operated in the intrapulse mode," Applied Physics B, vol. 103, no. 4, pp. 947-957, 2011.

[4] V. Ebert and J. Wolfrum, "Absorption spectroscopy," in Optical Measurements: Techniques and Applications, pp. 227-265, Springer, New York, NY, USA, 2001.

[5] G. Casa, D. A. Parretta, A. Castrillo, R. Wehr, and L. Gianfrani, "Highly accurate determinations of $\mathrm{CO}_{2}$ line strengths using intensity-stabilized diode laser absorption spectrometry," Journal of Chemical Physics, vol. 127, no. 8, Article ID 084311, 2007.

[6] C. Schulz, A. Dreizler, V. Ebert, and J. Wolfrum, "Combustion diagnostics," in Springer Handbook of Experimental Fluid Dynamics, C. Tropea, J. Foss, and A. Yarin, Eds., pp. 1241-1316, 2007.

[7] L. Mei, H. Jayaweera, P. Lundin, S. Svanberg, and G. Somesfalean, "Gas spectroscopy and optical path-length assessment in scattering media using a frequency-modulated continuouswave diode laser," Optics Letters, vol. 36, no. 16, pp. 3036-3038, 2011.

[8] D. Herriott, H. Kogelnik, and R. Kompfner, "Off-axis paths in spherical mirror interferometers," Applied Optics, vol. 3, no. 4, pp. 523-526, 1964.

[9] J. U. White, “Long optical paths of large aperture," Journal of the Optical Society of America, vol. 32, no. 5, p. 285, 1942.

[10] E. Flores, F. Idrees, P. Moussay et al., "Final report of the pilot study CCQM-P110-B1: A comparison of nitrogen dioxide $\left(\mathrm{NO}_{2}\right)$ in nitrogen standards at $10 \mu \mathrm{mol} / \mathrm{mol}$ by Fourier transform 
infrared spectroscopy (FT-IR)," Metrologia, vol. 49, Article ID 08006, 2012.

[11] GUM Workbench Professional Version 2.4 and GUM Workbench Manual for Version 2.4, Metrodata GmbH, Grenzach-Wyhlen, Germany, http://www.metrodata.de.

[12] J. A. Nwaboh, O. Werhahn, P. Ortwein, D. Schiel, and V. Ebert, "Laser-spectrometric gas analysis: $\mathrm{CO}_{2}$-TDLAS at $2 \mu \mathrm{m}$," Measurement Science and Technology, vol. 24, no. 1, Article ID 015202, 2013.

[13] B. Buchholz, N. Böse, and V. Ebert, "Absolute validation of a diode laser hygrometer via intercomparison with the German national primary water vapor standard," Applied Physics B, 2014.

[14] W. Gurlit, R. Zimmermann, C. Giesemann et al., "Lightweight diode laser spectrometer CHILD (compact high-altitude In-situ laser diode) for balloonborne measurements of water vapor and methane," Applied Optics, vol. 44, no. 1, pp. 91-102, 2005.

[15] A. Pogány, O. Ott, O. Werhahn, and V. Ebert, "Towards traceability in $\mathrm{CO}_{2}$ line strength measurements by TDLAS at $2.7 \mu \mathrm{m}$," Journal of Quantitative Spectroscopy and Radiative Transfer, vol. 130, pp. 147-157, 2013.

[16] O. Witzel, A. Klein, S. Wagner, C. Meffert, C. Schulz, and V. Ebert, "High-speed tunable diode laser absorption spectroscopy for sampling-free in-cylinder water vapor concentration measurements in an optical IC engine," Applied Physics B: Lasers and Optics, vol. 109, no. 3, pp. 521-532, 2012.

[17] S. Wagner, M. Klein, T. Kathrotia et al., "Absolute, spatially resolved, in situ CO profiles in atmospheric laminar counterflow diffusion flames using $2.3 \mu \mathrm{m}$ TDLAS," Applied Physics B: Lasers and Optics, vol. 109, no. 3, pp. 533-540, 2012.

[18] J. Henningsen and H. Simonsen, “The $\left(22^{0} 1-0^{0} 0\right)$ band of $\mathrm{CO}_{2}$ at $6348 \mathrm{~cm}^{-1}$ : linestrengths, broadening parameters, and pressure shifts," Journal of Molecular Spectroscopy, vol. 203, no. 1, pp. 1627, 2000.

[19] ISO Guide 98-3, Guide to the Expression of Uncertainty in Measurement, 1. International Organization for Standardization, Geneva, Switzerland, 2008, http://www.bipm.org/utils/common/documents/jcgm/JCGM_100_2008_E.pdf.

[20] Bundesanstalt für Materialforschung und- Prüfung (BAM), BLeast-software, also recommended by ISO 6143, http://www bam.de.

[21] ISO 6143: 2001, "Gas analysis-comparison methods for determining and checking the composition of calibration gas mixtures," 2001.

[22] EMRP-Project ENV06 (EUMETRISPEC), "Spectral reference data for atmospheric monitoring," http://www.eumetrispec.eu/.

[23] Origin 7.5 SR6, OriginLab Cooperation, Northampton, Mass, USA, 2006, http://www.OriginLab.com.

[24] L. S. Rothman, I. E. Gordon, A. Barbe et al., "HITRAN2008," Journal of Quantitative Spectroscopy and Radiative Transfer, vol. 110, pp. 533-572, 2009.

[25] N. Jacquinet-Husson, N. A. Scott, A. Chédin et al., “The GEISA spectroscopic database: current and future archive for earth and planetary atmosphere studies," Journal of Quantitative Spectroscopy and Radiative Transfer, vol. 109, no. 6, pp. 10431059, 2008.

[26] L. S. Rothman, N. Jacquinet-Husson, C. Boulet, and A. M. Perrin, "History and future of the molecular spectroscopic databases," Comptes Rendus Physique, vol. 6, no. 8, pp. 897-907, 2005.

[27] G. J. Padilla-Víquez, J. Koelliker-Delgado, O. Werhahn, K. Jousten, and D. Schiel, "Traceable $\mathrm{CO}_{2}-\mathrm{R}(12)$ line intensity for laser-spectroscopy-based gas analysis near $2 \mu \mathrm{m}$," IEEE Transactions on Instrumentation and Measurement, vol. 56, no. 2, pp. 529-533, 2007.

[28] G. Wübbeler, G. J. Padilla-Viquez, K. Jousten, O. Werhahn, and C. Elster, "Comparison and assessment of procedures for calculating the $\mathrm{R}(12)$ line strength of the $v_{1}+2 v_{2}+v_{3}$ band of $\mathrm{CO}_{2}$," Journal of Chemical Physics, vol. 135, no. 20, Article ID 204304, 2011. 

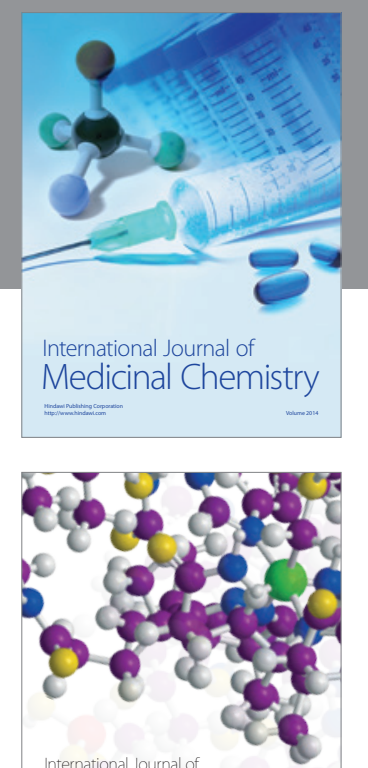

\section{Carbohydrate} Chemistry

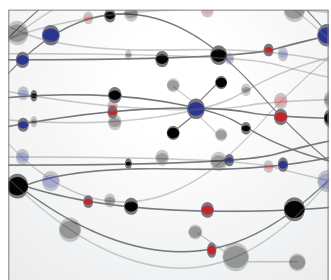

The Scientific World Journal
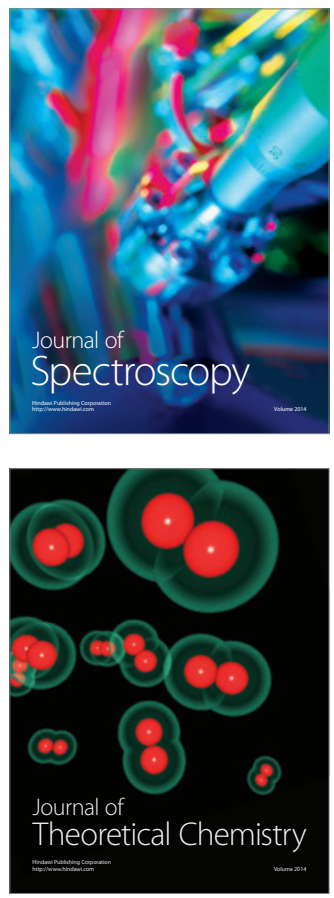
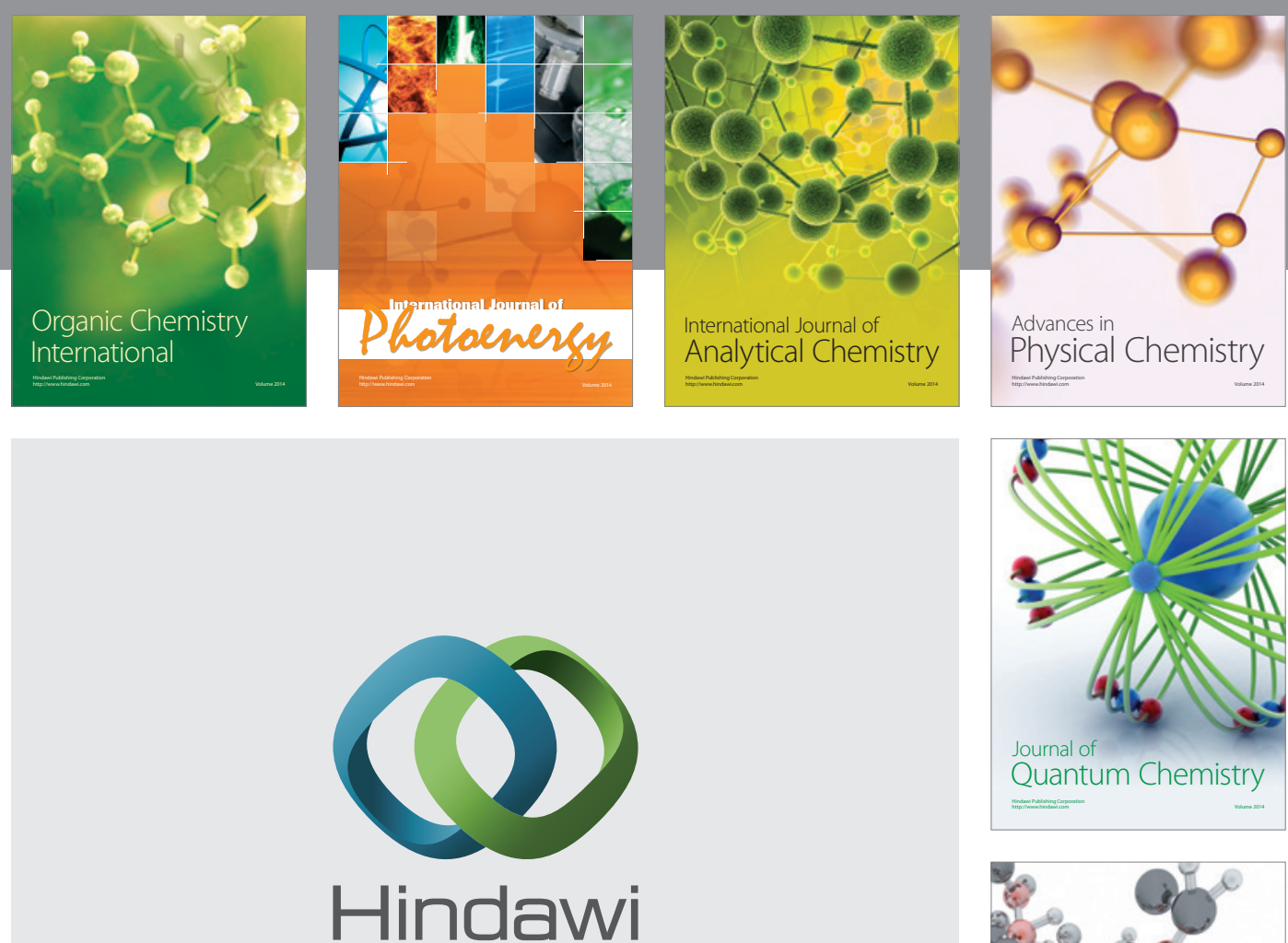

Submit your manuscripts at

http://www.hindawi.com

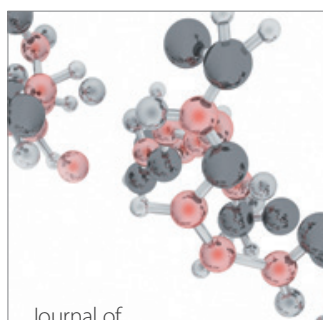

Analytical Methods

in Chemistry

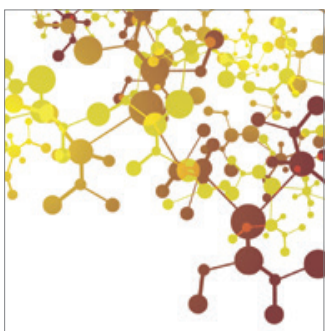

Journal of

Applied Chemistry

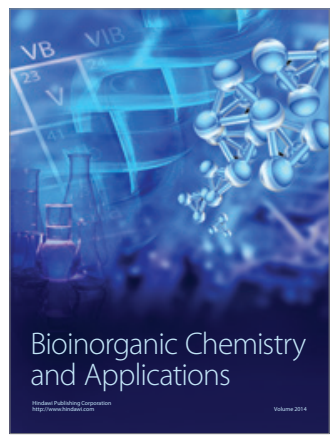

Inorganic Chemistry
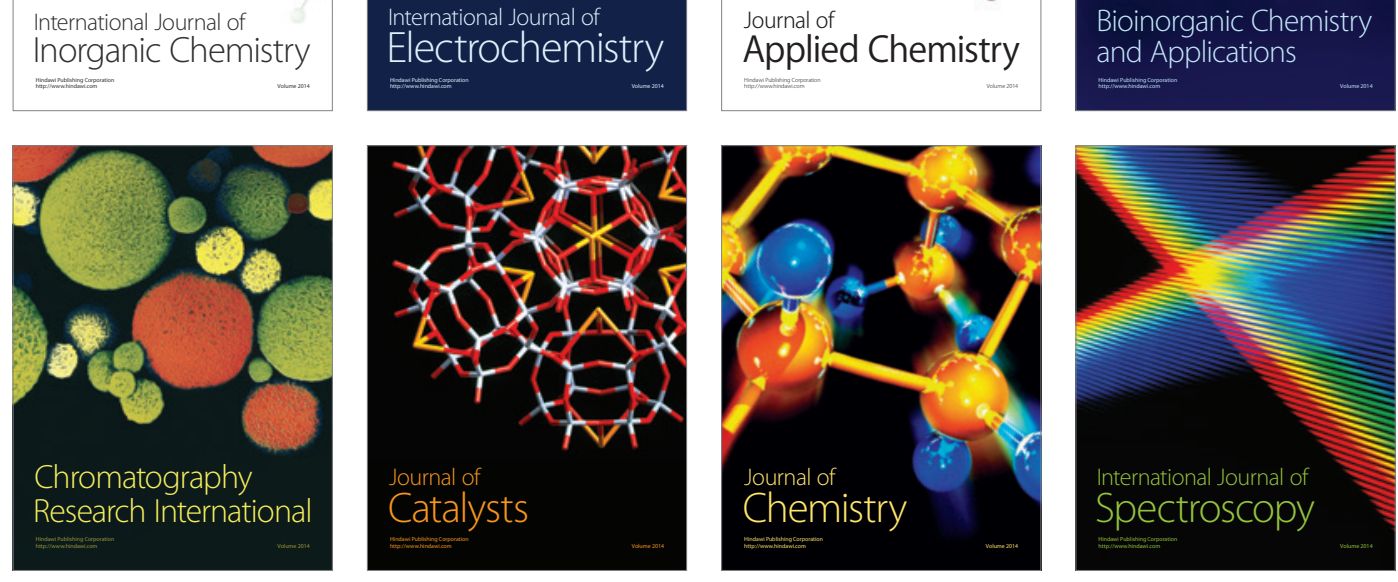